\title{
NOTES
}

\section{Proposals for Improving the Classification of Members of the Genus Shigella}

\author{
V. G. PETROVSKAYA' and N. A. KHOMENKO ${ }^{2}$ \\ Gamaleya Institute Ac. Med. Sc., Moscow D.98, ${ }^{\prime}$ and Mechnikov Institute of Vaccines and Sera, Moscow \\ $K-64,^{2}$ USSR
}

\begin{abstract}
With respect to Shigella flexneri, serovar 6 should be transferred to $S$. boydii; serovar 5 should be divided into two subserovars, $5 \mathrm{a}$ and $5 \mathrm{~b}$, with antigenic formulas V:(4) and V:7, respectively; and serovar 3 should contain only two subserovars, 3a-III:(3,4),6 and $3 \mathrm{~b}-\mathrm{III}:(3,4), 6,7,8$. Concerning the six provisional Shigella serovars, serovar 2000-53 should be removed from the genus because it belongs to the genus Escherichia, the position of serovar 1624-54 is still under consideration, serovars $3873-50$ and 3341-55 should be placed in $S$. dysenteriae as serovars 11 and 12 , respectively, and serovars $2710-54$ and 3615-53 should be considered as members of $S$. boydii serovars 16 and 17 , respectively.
\end{abstract}

In the past, many difficulties were encountered in classifying the members of the genus Shigella, and the problem of the classification of the infrasubspecific forms of $S$. flexneri is still under consideration. For example, scientists from different countries have indicated the necessity of removing serovar 6 from S. flexneri because its properties differ greatly from those of other members of this species. These differences include fermentative properties $(2,15)$, the range of susceptibility to phages and colicins, the immunochemical and immunoelectrophoretic characteristics of the antigens, and genetic characteristics $(4,5,8,12-15)$.

Recent immunochemical data have also shown the necessity of excluding serovar 6 from $S$. flexneri. The lipopolysaccharide of serovar 6 , contrary to the lipopolysaccharides of the other serovars of $S$. flexneri, contains $\mathrm{N}$-acetylgalactosamine instead of $\mathrm{N}$-acetylglucosamine (5), a sugar of the immunodeterminant group of antigens 3 and 4 (10-12). Significant genetic differences have also been demonstrated: serovar-specific antigen VI is controlled by the chromosomal gene located near the his marker (8). Antigens I through $V$ are the result of the modification of the basal lipopolysaccharide structure (antigens 3 and 4), which is controlled by the corresponding converting prophages localized near the lac marker $(8,9,14,15)$.

We believe that $S$. flexneri serovar 6 is close to $S$. boydii and that it should be transferred to this species. Ewing (2) placed it between $S$. flexneri serovars 1 through 5 and $S$. boydii. $S$. flexneri serovar 6 is closer to $S$. boydii than to $S$. flexneri on the basis of its biochemical properties (2), the percentage of colicinogenic strains, the type of colicins produced (14), and the immunochemical structure of its lipopolysaccharide (5). An analysis of the chemovars of $S$. boydii (1) permitted the determination of the position of serovar 6 as being intermediate between that of chemovars III and IV of $S$. boydii (8). It may be designated as $S$. boydii 18 , for instance (see below, concerning serovars 16 and 17). It is known that in $S$. boydii there is no correlation between serovars and the lipopolysaccharide chemotypes. The strains of the same chemotype do not give cross-reactions; the only serovars (10 and 11) which give such reactions belong to different chemotypes (1).

Supporting the division of serovar 5 into subserovars $5 \mathrm{a}$ and $5 \mathrm{~b}$ are the independent occurrence in nature of strains with the antigenic formula V:(4) or V: 7 (6) and differences in the genetic behavior of these strains $(8,9,14,15)$. This division has also been supported by the results of immunochemical investigations $(10$, $11,19)$.

Serovar 3 should also be reconsidered. As mentioned above, antigens 3 and 4 are the basic structures in the lipopolysaccharides of serovars 1 through 5 . Antigens 3 and 4 may be present in strains of any of the subserovars 1 through 5, resulting in the loss of the corresponding prophages. It seems irrational to increase the number of subdivisions of serovar 3 merely on the basis of a single variable character (the presence 
or absence of antigens 3 and 4) which distinguishes subserovar $3 b$ from $3 a(3)$. To unify the nomenclature of $S$. flexneri, it seems advisable to recognize only two subserovars of serovar 3 , $3 \mathrm{a}$ and $3 \mathrm{~b}$. Strains with the formula III: $(3,4), 6$ would be members of subserovar $3 \mathrm{a}$, and those with formula III:(3,4),6,7,8 would be members of subserovar $3 \mathrm{~b}(8)$. In this case, all subserovar "a" strains would be monolysogenic and would turn into variant "Y" after loss of the corresponding prophages. Strains of subserovar " $b$ " are bilysogenic and are characterized by more complicated variations in their antigenic structure (Fig. 1).

The proposed division may also be convenient in medical practice. Figure 1 may be used by epidemiologists in analyzing "change of types" observed in natural conditions in differentiating the above-mentioned antigenic variability from superinfection or mixed infections.

In summary, it is suggested that the classification of $S$. flexneri serovars be changed as follows (Table 1). (i) Serovar 6 should be removed from $S$. flexneri and transferred to $S$. boydii. (ii) Serovar 5 should be divided into two subserovars, $5 \mathrm{a}$ and $5 \mathrm{~b}$, with antigenic formulas V:(4) and V:7, respectively. (iii) Serovar 3 should be divided into only two subserovars, $3 \mathrm{a}$ and $3 \mathrm{~b}$, with formulas III:(3,4), 6 and III: $(3,4), 6,7,8$, respectively.

As for the so-called provisional serovars, one of them, 2000-53, should be excluded from Shigella because its fermentation pattern is typical of Escherichia. It grows on acetate agar, alkalinizes Christensen citrate and litmus milk, and is resistant to the phage which is specific for the genus Shigella (7). Thus, strains of serovar 2000 53 are anaerogenic, lactose-nonfermenting, nonmotile members of $E$. coli whose antigenic formula is $06 \mathrm{a}, 06 \mathrm{~b}: \mathrm{K}$ ?:H-. Strains of provisional serovars $3873-50$ and $3341-55$, which correspond to $S$. dysenteriae on the basis of their cultural and biochemical properties, should be regarded

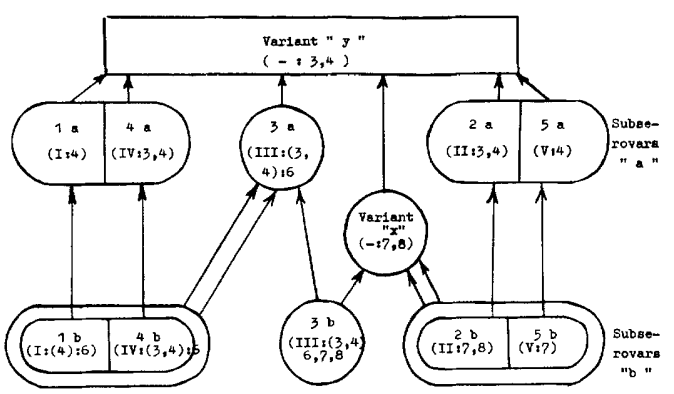

FIG. 1. Suggested relationships of S. flexneri serovars.
TABLE 1. Proposed classification of S. flexneri serovars

\begin{tabular}{cll}
\hline Serovar & Subserovar & $\begin{array}{c}\text { Antigenic } \\
\text { formula } \\
\text { (abbreviated) }\end{array}$ \\
\hline 1 & 1a & I:4 \\
& 1b & I:(4),6 \\
2 & $2 \mathrm{a}$ & II:3,4 \\
& $2 \mathrm{~b}$ & II: 7,8 \\
3 & $3 \mathrm{a}$ & III: $(3,4), 6$ \\
& $3 \mathrm{~b}$ & III: $(3,4), 6,7,8$ \\
4 & $4 \mathrm{a}$ & IV:(3,4) \\
& $4 \mathrm{~b}$ & $\mathrm{~V}:(4,4), 6$ \\
5 & $5 \mathrm{a}$ & $\mathrm{V}: 7$ \\
& $5 \mathrm{~b}$ & $-: 7,8$ \\
& var. X & $-: 3,4$ \\
\hline
\end{tabular}

as strains of serovars 11 and 12 , respectively, of S. dysenteriae. Provisional serovars 2710-54 and $3615-53$ should be regarded as serovars 16 and 17 , respectively, of $S$. boydii for the same reasons. The status of serovar 1621-54 should remain provisional until further strains can be collected and studied.

\section{REPRINT REQUESTS}

Address reprint requests to: Dr. V. G. Petrovska, Gamaleya Institute, Ac. Med. Sc., Moscow D-98, USSR.

\section{LTERATURE CITED}

1. Dmitriev, B. A., L. V. Backinowsky, N. K. Kochetkov, and N. A. Khomenko. 1973. Immunochemical studies on Shigella boydii lipopolysaccharides. Eur. J. Biochem. 34:513-518.

2. Ewing, W. H. 1974. Differentiation of Enterobacteriaceae by biochemical reactions. Public Health Service, Center for Disease Control, Atlanta, Georgia.

3. Ewing, W. H., and K. P. Carpenter. 1966. Recommended designations for the subserotypes of Shigella flexneri. Int. J. Syst. Bacteriol. 16:145-149.

4. Gekker, V. D., E. D. Ravitch-Birger, and Y. A. Belaya. 1965. The position of Newcastle bacteria in the classification of the shigellae. Int. Bull. Bacteriol. Nomencl. Taxon. 15:133-136.

5. Katzenellenbogen, E., M. Mulczyk, and E. Romanowska. 1976. Structural studies on $O$ specific polysaccharide of Shigella flexneri serotype 6. Eur. J. Biochem. 61:191-197.

6. Khomenko, N. A. 1967. Serological varieties of Shigella flexneri serotype 5. Int. J. Syst. Bacteriol. 17:53-55.

7. Khomenko, N. A. 1978. A study of a collection of the strains of Shigella provisional serovars. Z. Microbiol. (Moscow) 5:11-15.

8. Petrovskaya, V. G., and V. M. Bondarenko. 1977. Recommended corrections to the classification of Shi. gella flexneri on a genetic basis. Int. J. Syst. Bacteriol. 27:171-175.

9. Petrovskaya, V. G., and V. M. Bondarenko. 1971. The genetic control of Shigella flexneri type and group antigens. In Perez-Miravete and Pelars (ed.), Recent advances in microbiology. 10th Int. Congr. Microbiol. Mexico.

10. Seltmann, G. 1972. Untersuchungen zur Antigenstruktur von Shigellen. Z. Allg. Mikrobiol. 12:497-520. 
11. Seltmann, G. 1977. Shigella Immunochemistry. II. Z. Microbiol. (Moscow) 7:20-25.

12. Simmons, D. A. R. 1971. Immunochemistry of Shigella flexneri O-antigens: a study of structural and genetic aspects of biosynthesis of cell-surface antigens. Bacteriol. Rev. 35:117-148.

13. Slopek S., and M. Mulczyk. 1967. Concerning the classification of Shigella flexneri 6 bacilli. Arch. Immun.
Ther. Exp. 15:600-604.

14. Timakov, V. D., and V. G. Petrovskaya. 1972. Shigella flexneri biology and genetics. Medicina (Moscow).

15. Timakov, V. D., V. G. Petrovskaya, V. M. Bondarenko, and N. A. Khomenko. 1972. Genetic data concerning Shigella flexneri serotypes 5 and 6. Int. J. Syst. Bacteriol. 22:149-154. 